\title{
Microstructure and magnetic properties of very thin CoCr films deposited on different underlayers by rf-sputtering
}

\author{
G. Pan, D.J. Mapps, M.A. Akhter \\ School of Electronic, Communication and Electrical Engineering, Polytechnic South West, Plymouth, Deion, PL4 8.4A, UK
}

\author{
J.C. Lodder, P. ten Berge
}

Faculty of Electrical Engineering and Applied Physics, Unicersity of Twente, P.O. Box 217, 7500 AE Enschede. The Netherlands

\author{
H.Y. Wong and J.N. Chapman \\ Department of Physics and Astronomy, The Unicersity of Glasgow, Glasgow, G12 8QQ, UK
}

\begin{abstract}
Very thin $\mathrm{CoCr}$ films deposited on different underlayers on glass disk substrates were studied by the magneto-optic Kerr effect, VSM, torque magnetometry and TEM selected area diffraction. Square or near square perpericuicular loops were obtained from $\mathrm{Co} / \mathrm{Ti}, \mathrm{CoCr} / \mathrm{Au}, \mathrm{CoCr} / \mathrm{Al}, \mathrm{CoCr} / \mathrm{C}$ and $\mathrm{CoCr} / \mathrm{Si}$ films. TEM SAD study revealed that the crystalline structure is a key factor determining the magnetic anisotropy of the very thin CoCr films. In particular, the $c$-axis of the hcp $\mathrm{CoCr}$ filins which exhibit square perpendicular loops is perpendicular to the film plane whilst that of the CoCr films which exhibit a thin and flat perpendicular loop lies in the film plane. The texture of the very thin CoCr films deposited on different underlayers is mainly dependent on the structure and texture of underlayers. The relation between the structure of $\mathrm{CoCr}$ and its underlayers is discussed.
\end{abstract}

\section{Introduction}

There is a considerable current interest in the effect of underlayers on the magnetic properties of both perpendicular and longitudinal magnetic recording media [1,2]. In a previous paper [1] we have reported on the magnetic properties of very thin $\mathrm{CoCr}$ films deposited on $\mathrm{Ti}$ underlayers. Very pronounced effects of Ti underlayers on the shearing and the squareness ratio of the perpendicular loops have been observed in such $\mathrm{CoCr}$ films. In order to understand the origin of the underlayer effect, further experimental investigation into the microstructure, micromagnetics and magnetic properties of these films have been un-

Correspondence to: Dr. G. Pan, School of Electronic, Communication and Electrical Engineering. Polytechnic South West, Plymouth, Devon, PL4 8AA, UK. dertaken. We report here on a detailed experimental programme of magnetic measurements together with microstructural examinations by TEM of the $160 \AA$ thick $\mathrm{CoCr}$ films with different underlayers.

\section{Experimental details}

Very thin CoCr films were deposited on different underlayers or directly on to glass disk substrates. The film thickness was measured by a talystep and a Scanning Auger microprobe (SAM). The $\mathrm{Cr}$ content of the $\mathrm{CoCr}$ films is 23 at $\%$ by Auger. Trae magnetic pioperties of the $\mathrm{CoCr}$ films were studied using a magneto-optic Kerr effect (MOKE) system, a, vibration sample magnetometer (VSM) and a torque magnetometer. The film structure and crystal orientation 


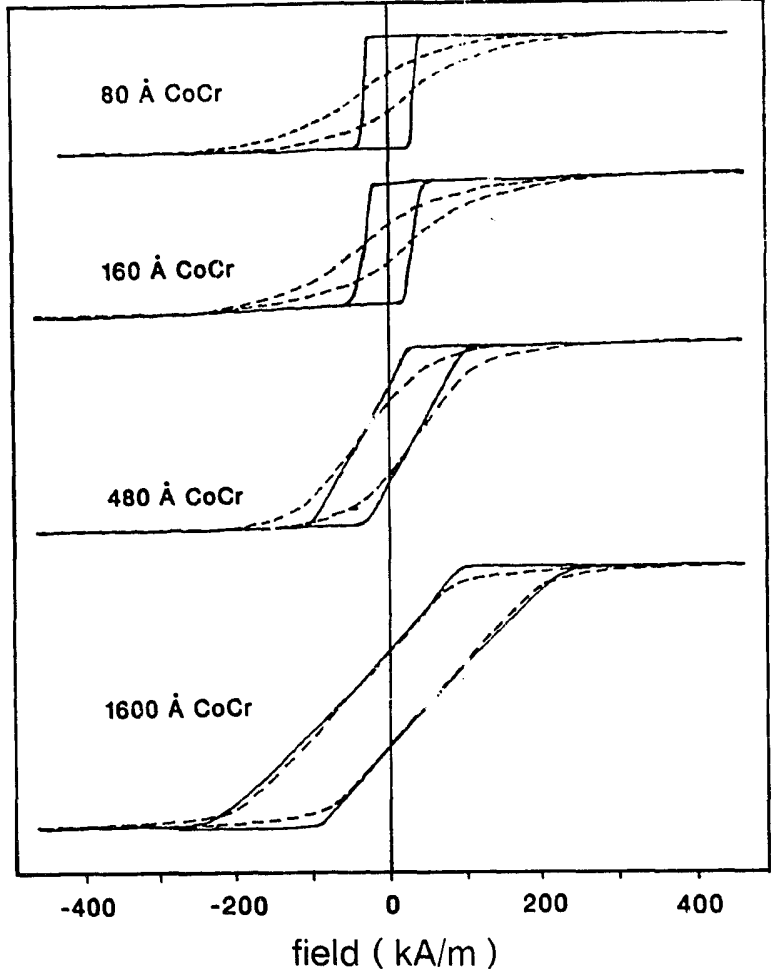

Fig. 1. Perpendicular MOKE loops of different thickness $\mathrm{CoCr}$ films with and without $\mathrm{Ti}$ underlayers (solid and dashed line respectively). Vertical axis is in arbitrary units.

were investigated by selected area diffraction (SAD) on a JEOL 2000FX TEM. For the preparation of TEM specimens, glass disk substrates were precoated with carbon films before the deposition of underlayers and the $\mathrm{CoCr}$ films themselves. The films were then floated off in water.

\section{Results}

\subsection{Magnetic measurements}

Fig. 1 shows the perpendicular MOKE loops of different thickness CoCi films with Ti underlayers (solid line) and without $\mathrm{Ti}$ underlayers (dashed line). For the $1600 \AA \AA$ thick CoCr films. the principal difference of the MOKE loops of $\mathrm{CoCr}$ films with and without $\mathrm{Ti}$ underlayers is only in the "shoulder" of the loop. As the $\mathrm{CoCr}$ film thickness reduces, the MOKE loops of the $\mathrm{CoCr}$ films deposited directly on glass become more rounded. On the other hand, the MOKE loops of $\mathrm{CoC}$ : films on $\mathrm{Ti}$ underlayers become steeper and squarer. The sliearing of the perpendicular loops has been discussed in the light of a stripe domain model suggested by Weilinga and Lodder [3] and a particulate model suggested by Chang and Fredkin $[1,4]$.

Fig. 2 shows the Ti underlayer thickness effect on the perpendicular loops of $160 \AA$ thick $\mathrm{CoCr}$ films, which indicates that the squareness and the shearing of the loops are strongly affected by the existence of even a very thin Ti underlayer. The minimum $\mathrm{Ti}$ thickness to obtain a square loop is about $250 \AA$.

The perpendicular $M-H$ loops of $160 \AA$ thick $\mathrm{CoCr}$ films without $\mathrm{Ti}$ underlayer and with $\mathrm{Ti}$ underlayer were also measured by VSM and results are shown in fig. 3. The square VSM perpendicular loop for the $160 \AA$ thick CoCr film on $\mathrm{Ti}$ and $\mathrm{a}$ thin and curved one for the films without $\mathrm{Ti}$ are in good agreement with the corre-

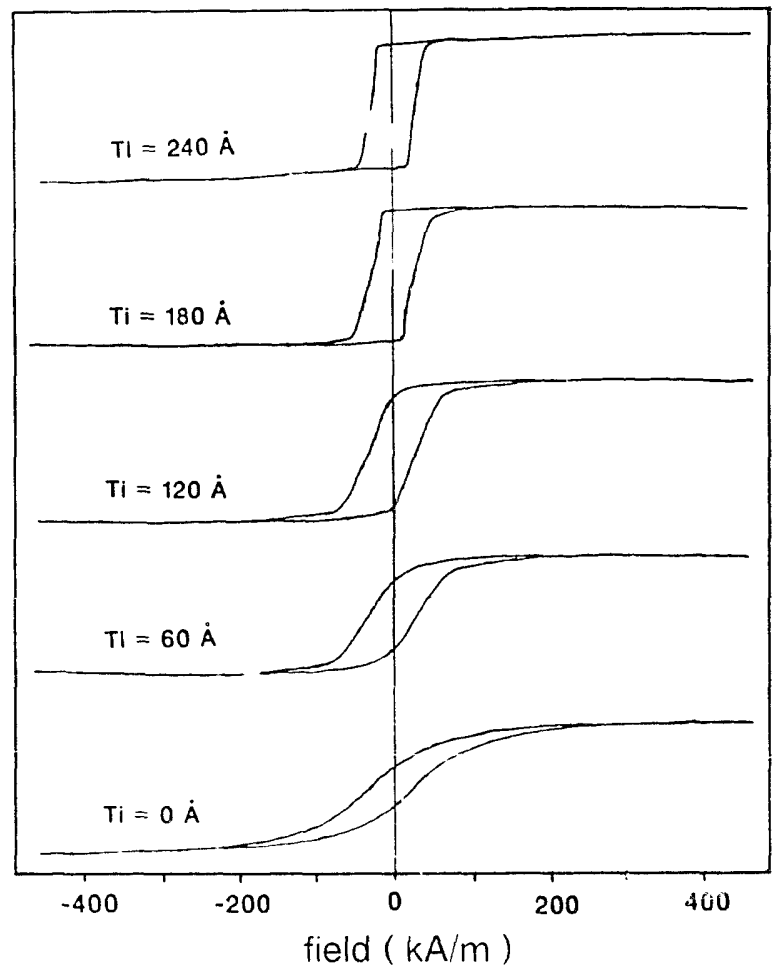

Fig. 2. Ti underlayer thickness effect on the perpendicular MOKE loops of $160 \AA \AA$ thick CoCr films. Vertical axis is in arbitrary units. 

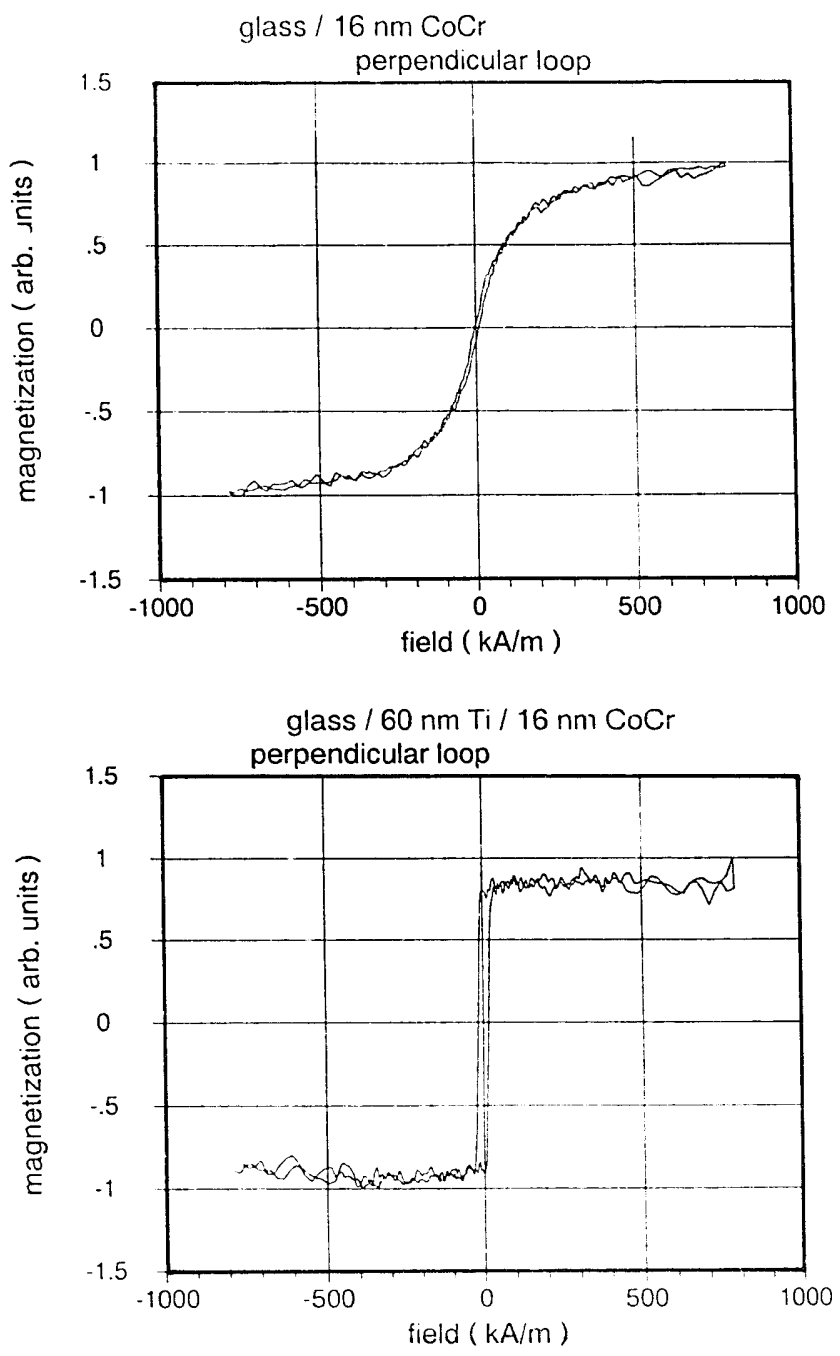

Fig. 3. $M-H$ loops of $160 \AA$ thick $\mathrm{CoCr}$ films with and without $\mathrm{Ti}$ underlayers measured by VSM.

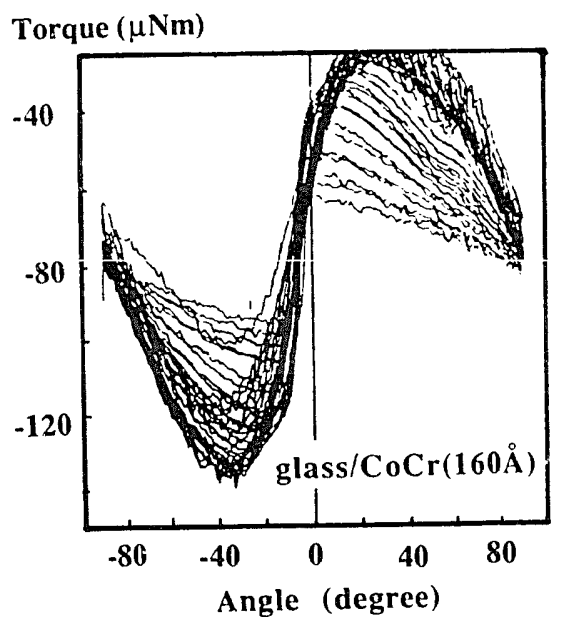

sponding MOKE loops, indicating an improved perpendicular easy axis behavior of the $\mathrm{CoCr}$ films gown on Ti underlayers.

Fig. 4 shows the torque magnetometer plots of the $160 \AA$ thick $\mathrm{CoCr}$ films with and without $\mathrm{Ti}$ underlayers. Torque curves for both samples were initiated with the applied field in the plane of the sample. Under this condition, torque curves which start at zero degree with negative slope indicate an anisotropy perpendicular to the film plane and with a positive slope indicate an anisotropy in the plane of the films. As shown in fig. 4, the $\mathrm{CoCr}$ on giass exhibits a typical in-plane anisotropy torque curve, while the $\mathrm{CoCr}$ film on $\mathrm{Ti}$ exhibit a near perpendicular anisotropy torque curve. It is obvious that the magnetization easy axis of such thin $\mathrm{CoCr}$ film is dependent on the $\mathrm{Ti}$ underlayer.

Further experiment on the very thin $\mathrm{CoCr}$ films with other different underlayers was undertaken and fig. 5 shows the perpendicular MOKE loops of $80 \AA$ thick $\mathrm{CoCr}$ films on different underlayers. Among them, the $80 \AA$ thick $\mathrm{CoCr}$ films on $\mathrm{Ti}, \mathrm{Au}, \mathrm{Al}$ and $\mathrm{C}$ underlayers or directly deposited on Si substrate exhibit square or near square perpendicular MOKE loops. By contrast the $\mathrm{CoCr}$ film on a $\mathrm{Cr}$ underlayer exhibits a thin and flat perpendicular loop. The different height of the vertical axis of the loops is believed to be caused by the underlayer enhancement [5]. The effect of different underlayers on the magnetic properties of the initial growth layer of $\mathrm{CoCr}$ films can be clearly seen from this figure.

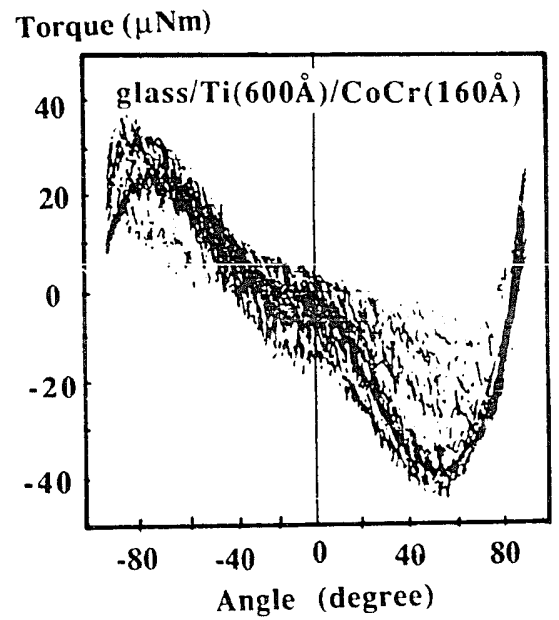

Fig. 4. Torque curves of $160 \AA$ thick $\mathrm{CoCr}$ films with and without Ti underlayers. 

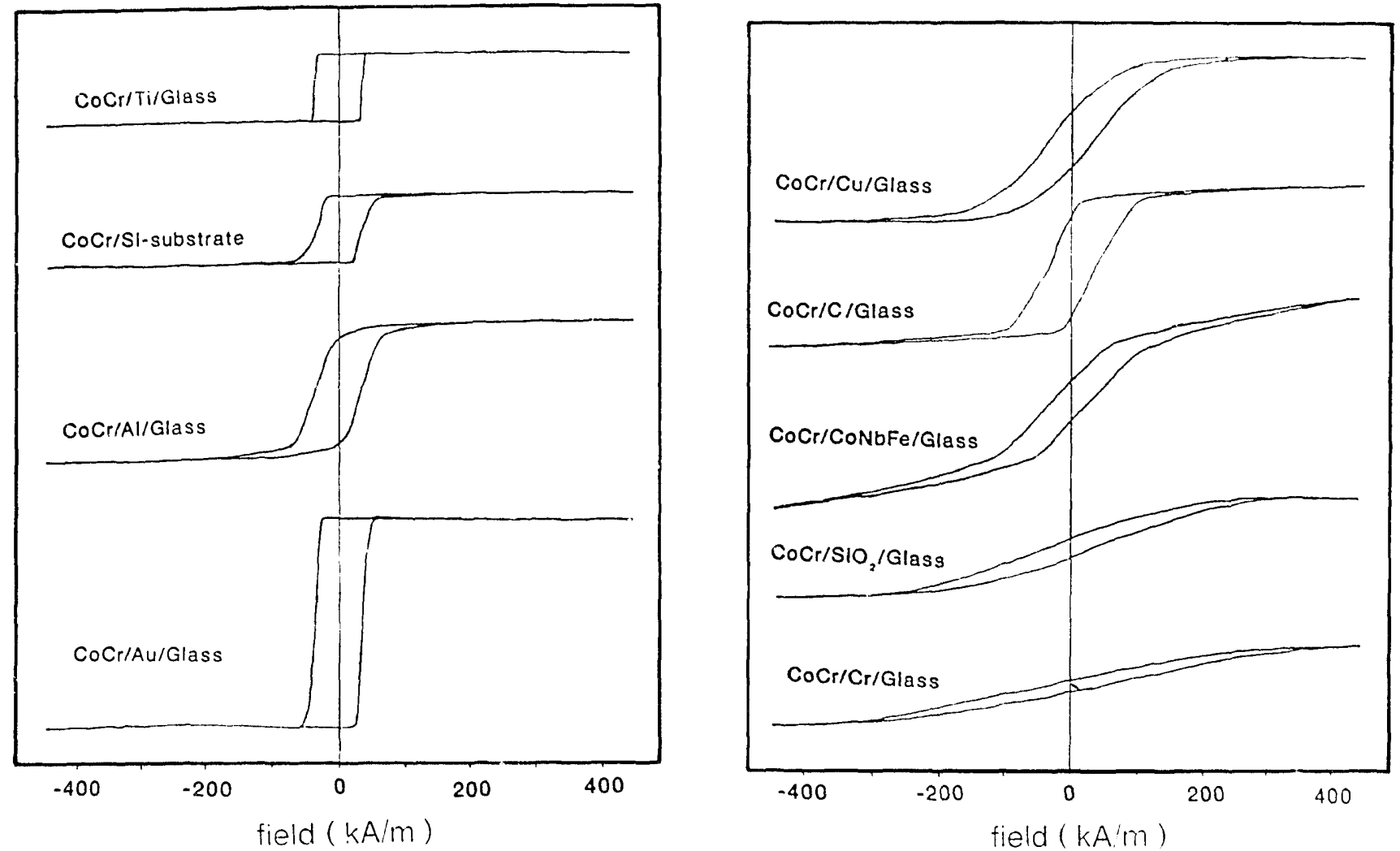

Fig. 5. Perpendicular MOKE loops of $80 \AA$ A thick CoCr films deposited on different underlayers, the $Y$ axis is in arbitrary units, but in the same scaling.

\subsection{Film microstructure and texture}

In order to understand the structural origin of the underlayer effect, the $\mathrm{CoCr}$ films and their underlayers were studied by observing selected area diffraction (SAD) patterns with the specimen untilied and tilted through $30^{\circ}$ on a JEOL 2000FX TEM.

Let us start with a brief review of the electron diffraction techniques for polycrystalline thin films [6]. In electron diffraction, only those planes which are near parallel to the incident electron beam contribute to the diffraction patterns because the Bragg angle in electron diffraction is very small (less than $1^{\circ}$ or $2^{\circ}$ ).

Fig. 6a is a schematic drawing of a [00.1] oriented hcp Co crystal and the SAD pattern of the [00.1] textural hep Co films. For such films, the main reflection rings are $(1 \overline{1} 00),(11 \overline{2} 0),(20 \overline{2} 0)$, $\ldots$, all of which are in the form of $\{h k i 0\}$. Reflec- tion rings of $(0002),(01 \overline{1} 1), \ldots,\{$ hkil $\}(l \neq 0)$ would not appear. When the specimen is tilted through $30^{\circ}$, the $\{h k i 0\}$ rings break into arcs along the diameter parallel to the tilting axis; along the perpendicular diameter, arcs such as (0111) appear because this plane makes an angle of $28^{\circ}$ with the $c$-axis. The schematic drawing of SAD patterns of the [11.0] and [10.0] textural Co films are shown in figs. $6 \mathrm{~b}$ and $\mathrm{c}$ respectively. When we observe the SAD patterns of the [11.0] and [10.0] textural polycrystalline films with specimen tilted, it is very difficult to predict when ares will occur for every family of planes because of the complex of angles within each family. However, one of the obvious features for surch textural films is that the $(0002)$ ring will break into arcs along the diameter parallel to the tilting axis.

The experimental resu'ts of the SAD patterns of $160 \AA$ thick $\mathrm{CoCr}$ films on $\mathrm{Ti}, \mathrm{Cr}, \mathrm{C}$ and $\mathrm{Cu}$ underlayers with the specimen untilted and tilted 


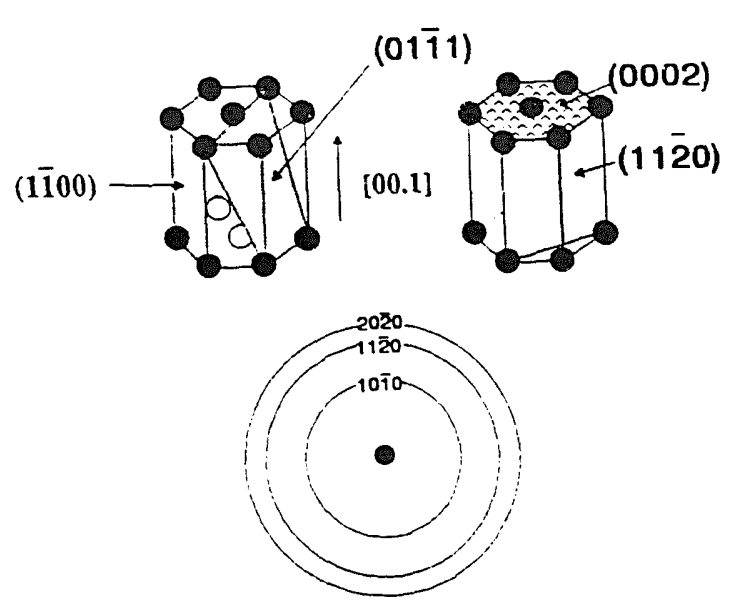

(a)

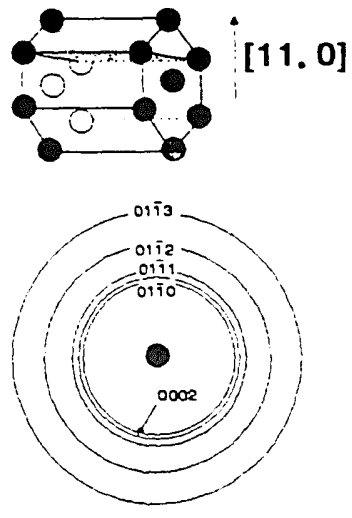

(b)
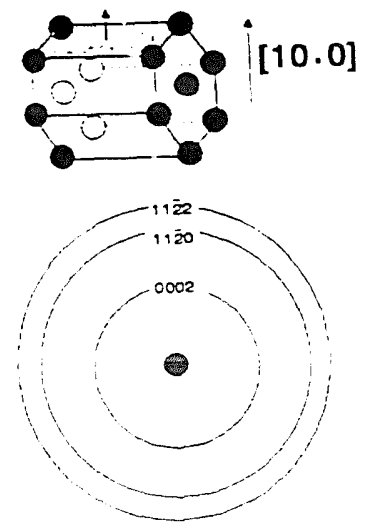

(c)
Fig. 6. Schematic drawing of (a) [00.1] zone axis SAD pattern, (b) [11.0] zone axis SAD pattern and (c) [10.0] zone axis SAD pattern of hcp Co polycrystalline films. through $30^{\circ}$ ar z shown in fig. 7. As mentioned above, these TEM specimens were deposited on a precoated $C$ layers on glass substrates. However, MOKE measurement of these films showed simiiar loops to those shown in fig. 5. The SAD patterns are indexed in tables 1 to 4 . If we examine figs. 6,7 and tables 1 to 4 carefully, the following conclusions can be drawn.

The SAD patterns of the CoCr films on $\mathrm{Ti}$ and $C$ underlayers with the specimen untilted and tilted are as expected from a strong [00.1] textural hcp films. The main reflection rings of the Co on both patterns are $(1 \overline{1} 00),(11 \overline{2} 0)$ and $(20 \overline{2} 0)$ when untilted. Diffraction rings of the form $(000 l)$, where $l \neq 0$, are not seen. When the specimen was tilted through $30^{\circ}$, the $(1 \overline{1} 00)$, (1120) and $(20 \overline{2} 0)$ rings are broken into arcs along the diameter parallel to the tilting axis. Arcs, such as (0111), appear along the perpendicular diametei. The $c$-axis of such films is perpendicular to the film plane.

The $\mathrm{CoCr}$ on $\mathrm{Cr}$ showed a mixed [11.0] and [10.0] zone axis patterns. When the specimen was tilted, the (0002) ring breaks into arcs. The $c$-axis of such films lies in the film plane. The $\mathrm{CoCr}$ on $\mathrm{Cu}$ has no texture. Reflection rings from all planes are present on the SAD patierns. When specimen was tilted, no changes to the pattern could be seen.

The underlayer structure can also be clarified from these patterns. The $\mathrm{Ti}$ underlayer has a

Table 1

Summary of SAD patterns of $\mathrm{C} / \operatorname{Ti}(300) \AA) / \operatorname{CoCr}(160 \AA)$ films with specimen untilted and tilted

\begin{tabular}{|c|c|c|c|c|c|c|c|}
\hline \multirow[t]{2}{*}{ Ring } & \multirow[t]{2}{*}{ Diameter } & \multicolumn{2}{|c|}{ Plane spacing $[\AA]$} & \multirow[t]{2}{*}{ Structure } & \multirow[t]{2}{*}{ hkil } & \multirow[t]{2}{*}{ Untilted } & \multirow[t]{2}{*}{ Tilted } \\
\hline & & measured & ASTM & & & & \\
\hline 1 & 29.0 & 2.544 & 2.555 & Ti hep & $10 \overline{10}$ & medium ring & ring $\&$ arcs $\|$ \\
\hline 2 & 31.5 & 2.342 & 2.342 & Ti hcp & 0002 & medium ring & unchanged \\
\hline 3 & 32.9 & 2.242 & 2.243 & Ti hcp & $01 \overline{1} 1$ & very weak ring & strong arcs $\perp$ \\
\hline 4 & 34.0 & 2.170 & 2.170 & Cohcp & $10 \overline{10}$ & strong ring & strong arcs! \\
\hline 5 & 38.5 & 1.916 & 1.915 & Co hcp & $01 \overline{1} 1$ & invisible & strong arcs $\perp$ \\
\hline 6 & 42.9 & 1.72 & 1.72 & Ti hcp & $01 \overline{1} 2$ & invisible & weak $\operatorname{arcs} \perp$ \\
\hline 7 & 50.2 & 1.470 & 1.475 & Ti hcp & $11 \overline{2} 0$ & medium ring & medium arcs\| \\
\hline 8 & 55.2 & 1.337 & 1.332 & Ti hcp & $01 \overline{1} 3$ & very weak ring & unchanged \\
\hline 9 & 58.6 & 1.259 & 1.253 & Co hcp & $11 \overline{2} 0$ & strong ring & strong arcs $\|$ \\
\hline 10 & 67.5 & 1.093 & 1.085 & Co hcp & $20 \overline{2} 0$ & very weak ring & weak ring \& arcs $\|$ \\
\hline
\end{tabular}


Table 2

Summary of SAD patterns of $\mathrm{C} / \mathrm{Cr}(300 \AA) / \operatorname{CoCr}(160 \AA)$ films with specimen untilted and tilted

\begin{tabular}{cllllllll}
\hline Ring & Diameter & \multicolumn{2}{l}{ Plane spacing $[\AA]$} & & Structure & hkil & Untilted & Tilted \\
\cline { 3 - 7 } & & measured & ASTM & & & & \\
\hline 1 & 34.0 & 2.170 & 2.170 & Co hcp & $10 \overline{1} 0$ & weak ring & unchanged \\
2 & 36.2 & 2.04 & 2.04 & Cr bcc & 011 & strong ring & ring \& arcs $\|$ \\
3 & 36.2 & 2.038 & 2.035 & Co hcp & 0002 & strong ring & ring \& arcs $\|$ \\
4 & 38.5 & 1.916 & 1.915 & Co hcp & $01 \overline{1} 1$ & strong ring & ring \& arcs $\|$ \\
5 & 49.8 & 1.481 & 1.484 & Co hcp & $01 \overline{1} 2$ & weak ring & weak arcs \\
6 & 51.0 & 1.447 & 1.443 & Cr bcc & 002 & strong ring & strong arcs $\|$ \\
7 & 58.6 & 1.259 & 1.253 & Co hcp & $11 \overline{2} 0$ & weak ring & unchanged \\
8 & 62.4 & 1.182 & 1.178 & Cr bcc & 112 & weak ring & medium arcs $\perp$ \\
9 & 63.8 & 1.156 & 1.15 & Co hcp & $01 \overline{1} 3$ & weak ring & unchanged \\
10 & 69 & 1.07 & 1.067 & Co hcp & $11 \overline{2} 2$ & very weak ring & unchanged \\
11 & 72 & 1.02 & 1.02 & Cr bcc & 022 & weak ring & ring $\&$ arcs $\|$ \\
\hline
\end{tabular}

Table 3

Summary of SAD patterns of $\mathrm{C} / \mathrm{CoCr}(160 \AA)$ films with specimen untilted and tilted

\begin{tabular}{|c|c|c|c|c|c|c|c|}
\hline \multirow[t]{2}{*}{ Ring } & \multirow[t]{2}{*}{ Diameter } & \multicolumn{2}{|c|}{ Plane spacing $[\AA ̊]$} & \multirow[t]{2}{*}{ Structure } & \multirow[t]{2}{*}{ hkil } & \multirow[t]{2}{*}{ Unitilted } & \multirow[t]{2}{*}{ Tilted } \\
\hline & & measured & $\overline{\mathrm{ASTM}}$ & & & & \\
\hline 1 & 27 & 2.17 & 2.17 & Co hcp & $10 \overline{10}$ & strong ring & strong arcs $\|$ \\
\hline 2 & 28.8 & 2.034 & 2.035 & Co hcp & 0002 & faint & faint \\
\hline 3 & 30.5 & 1.92 & 1.915 & Co hcp & $01 \overline{1} 1$ & faint & strong arcs $\perp$ \\
\hline 4 & 46.6 & 1.257 & 1.253 & Co hcp & $11 \overline{2} 0$ & strong ring & strong arcs $\|$ \\
\hline 5 & 53.8 & 1.089 & 1.085 & Co hcp & $20 \overline{2} 0$ & medium ring & medium arcs $\|$ \\
\hline 6 & 55 & 1.065 & 1.068 & Co hcp & $11 \overline{2} 2$ & faint & strong arcs $\perp$ \\
\hline 7 & 61.2 & 0.957 & 0.957 & Co hcp & $02 \overline{2} 2$ & invisable & weak arcs $\perp$ \\
\hline 8 & 71.6 & 0.818 & 0.82 & Co hcp & $12 \overline{3} 0$ & weak ring & medium arcs $\|$ \\
\hline
\end{tabular}

Table 4

Summary of SAD patterns of $\mathrm{C} / \mathrm{Cu}(300 \AA) / \operatorname{CoCr}(160 \AA)$ films with specimen untilted and tilted

\begin{tabular}{|c|c|c|c|c|c|c|c|}
\hline \multirow[t]{2}{*}{ Ring } & \multirow[t]{2}{*}{ Diameter } & \multicolumn{2}{|c|}{ Plane spacing [Ă] } & \multirow[t]{2}{*}{ Structure } & \multirow[t]{2}{*}{ hkil } & \multirow[t]{2}{*}{ Untilted } & \multirow[t]{2}{*}{ Tilted } \\
\hline & & measured & ASTM & & & & \\
\hline 1 & 34.0 & 2.170 & 2.170 & Co hcp & $10 \overline{10}$ & weak ring & unchanged \\
\hline 2 & 35.3 & 2.09 & 2.087 & $\mathrm{Cu} \mathrm{fcc}$ & 111 & strong ring & unchanged \\
\hline 3 & 36.0 & 2.049 & 2.038 & Co hcp & 0002 & medium ring & unchanged \\
\hline 4 & 38.5 & 1.916 & 1.915 & Co hcp & $01 \overline{1} 1$ & weak ring & unchanged \\
\hline 5 & 41.0 & 1.80 & 1.808 & $\mathrm{Cu} \mathrm{fcc}$ & 002 & medium ring & unchanged \\
\hline 6 & 57.8 & 1.276 & 1.278 & $\mathrm{Cu} \mathrm{fcc}$ & 022 & medium ring & unchanged \\
\hline 7 & 58.6 & 1.259 & 1.253 & Co hcp & $11 \overline{2} 0$ & medium ring & unchanged \\
\hline 8 & 68.0 & 1.085 & 1.085 & Co hcp & $20 \overline{2} 0$ & medium ring & uncharged \\
\hline 9 & 69.4 & 1.063 & 1.067 & Co hcp & $11 \overline{2} 2$ & weak ring & unchanged \\
\hline 10 & 71.0 & 1.039 & 1.044 & $\mathrm{Cufcc}$ & 222 & weak ring & unchanged \\
\hline
\end{tabular}

preferred hcp [00.1] texture. The $\mathrm{C}$ layer is amorphous. The $\mathrm{Cr}$ underlayer has a preferred bcc [100] texture. The $\mathrm{Cu}$ underlayer has a fcc struc- ture with no texture. The correlation of the underlayer structure and the $\mathrm{CoCr}$ texture is schematically given in fig. 8 . 
specimen untilted

specimen tilted $30^{\circ}$
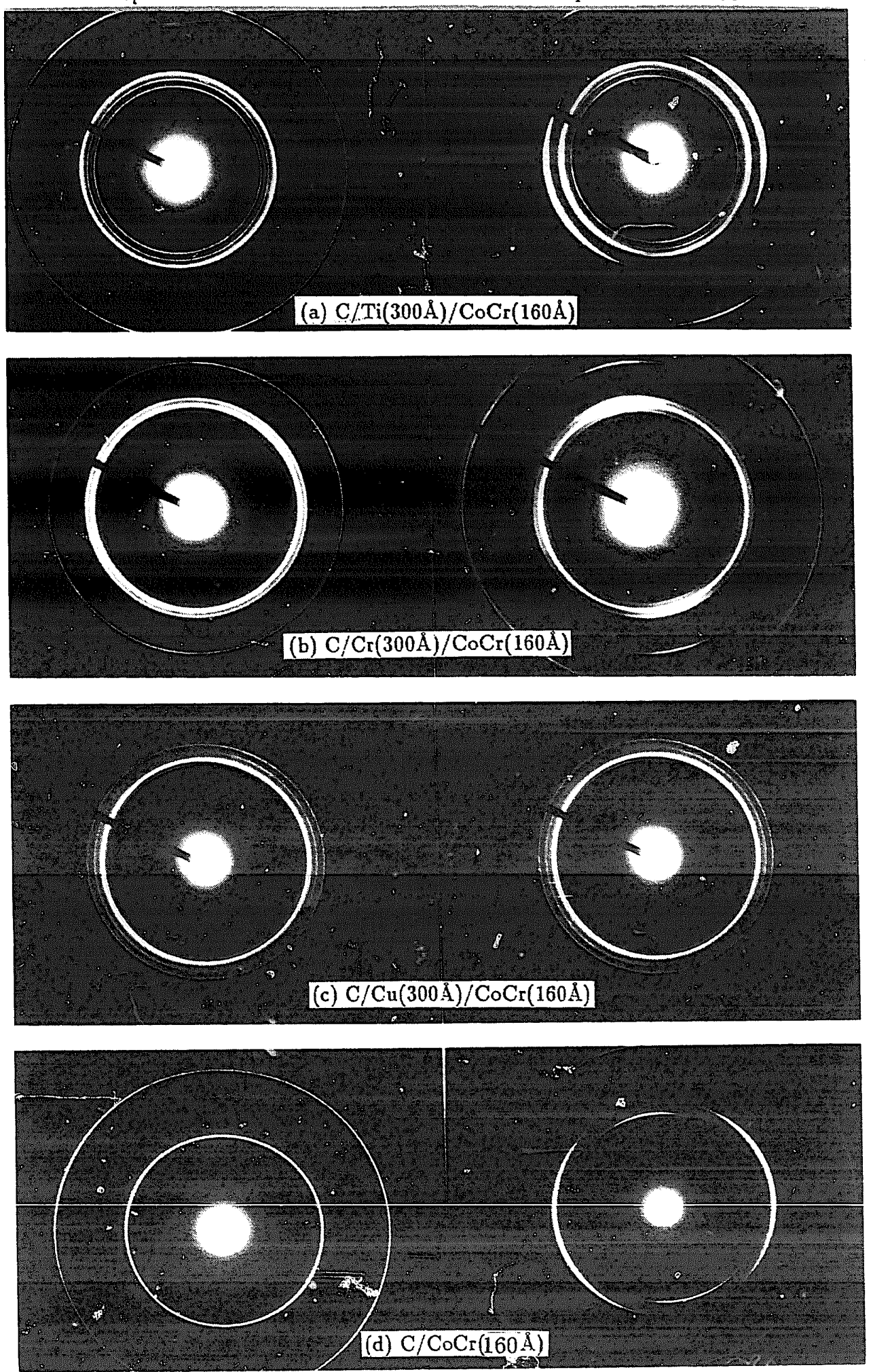

Fig. 7. SAD patterns of $160 \AA$ thick $\mathrm{CoCr}$ films dep, itca on $\mathrm{Ti}, \mathrm{Cr}, \mathrm{Cu}$ and $\mathrm{C}$ underlayers. 

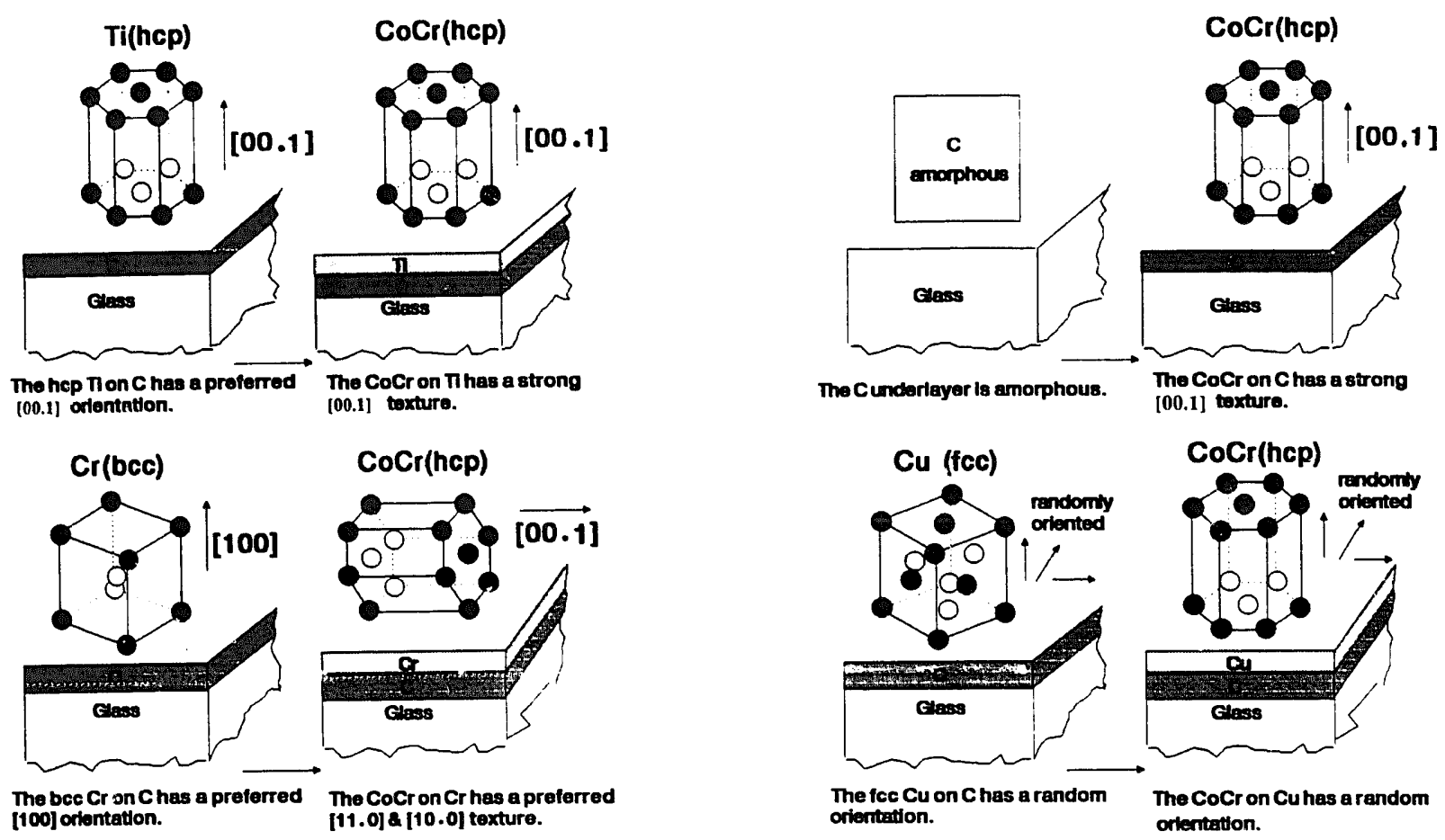

Fig. 8. Schematic representation of the effect of underlayer strecture on the texture on $\mathrm{CoCr}$ films.

\section{Discussion and conclusions}

In summary, we conclude that the square or near square perpendicular loops of the very thin $\mathrm{CoCr}$ films originate from the improved perpendicular anisotropy of the films due to their growth on appropriate underlayers. Our TEM SAD study revealed that the microstructure is a key factor in determining the easy axis anisotropy of the very thin $\mathrm{CoCr}$ films. The $c$-axis of those hcp $\mathrm{CoCr}$ films which exhibit square perpendicular loops, such as $\mathrm{CoCr} / \mathrm{Ti}$ and $\mathrm{CoCr} / \mathrm{C}$, are perpendicular to the film plane. The $c$-axis of the hcp $\mathrm{CoCr}$ films which exhibit poor perper. .lcular loops, such as $\mathrm{CoCr} / \mathrm{Cr}$, lies in the film plane.

The texture of the very thin $\mathrm{CoCr}$ films deposited on different underlayers is dominated by the structure and texture of the underlayers. The Ti underlayer which has a preierred hcp [00.1] texture encourages the hcp $\mathrm{CoCr}$ to grow epitaxially into a strong $c$-axis axture. The amorphous $\mathrm{C}$ is another suitable underlayer for the growth of [00.1] textural CoCr films. This may be explained by the fact that the $\mathrm{CoCr}$ film itself has the potential to grow into a $c$-axis texture because its
(0002) plane has the lowest surface energy [2], and the amorphous $\mathrm{C}$ underlayer provides a free-growth substrate condition for the $\mathrm{CoCr}$. The $\mathrm{CoCr}$ films on the [100] textural bcc $\mathrm{Cr}$ underlayer were forced to grow into a mixed [11.0] and [10.0] texture. This is perhaps due to the bcc [100] texture being more suitable for the epitaxial growth of hcp CoCr films with $c$-axis lying in the film plane.

\section{References}

[1] D.J. Mapps, M.A. Akhter and G. Pan, IEEF Trans. Magn. MAG-26 (1990) 1614.

[2] T. Yeh, J.M. Sivertsen and J.H. Judy, IEEE Trans. Magn. MAG-26 (1990) 1590.

[3] T. Wielingaa and J.C. Lodder, Phys. Stat. Sol. (a) 96 (1986) 255 .

[4] Ching-Ray Chang and D.R. Fredkin, IEEE Trans. Magn. MAG-23 (1987) 2052.

[5] J. Zak, E.R. Moog, C. Liu and S.D. Bader, J. Magn. Magn. Mater. 89 (1990) 107.

[6] J.W. Edington, Electron diffraction in the electron microscope, Philips (1975). 